Preface

\title{
Advances in Autism Spectrum Disorder
}

\author{
Paolo Curatolo ${ }^{1}$ Luigi Mazzone ${ }^{1}$ \\ ${ }^{1}$ Child Neurology and Psychiatry Unit, System Medicine Department, \\ Tor Vergata, University Hospital of Rome, Italy \\ J Pediatr Neurol 2017;15:95.
}

The recent increase in autism spectrum disorder (ASD) prevalence highlights the need for continued efforts to translate novel ASD discoveries into effective interventions. On the basis of the recent advanced genome analysis techniques, several genes associated with ASD have been identified, which are genes encoding proteins associated with the synaptic function. Many different genes are linked together in gene networks that seem to converge to a relatively limited number of biological pathways.

To move forward toward the goal of understanding all of the genetic factors involved it is necessary to scan the genome in its entirety using whole genome sequencing on thousands of samples.

However, because there is no reason to suspect that mutation rates have increased suddenly in a short period of time, the increase in the incidence of ASD cannot be explained only by genetic factors; therefore, the interactions with environmental factors should be considered.

Despite considerable progress in understanding the neurobiology of ASD, established treatments for core symptoms are still needed. An early behavioral intervention is critical for improving the long-term outcomes, however, to date, pharmacological treatments for core symptoms of ASD are still lacking.

The wide variety of genetic variants present in ASD that converge in different molecular and biochemical pathways mediating the phenotypic expression in some identifiable core symptoms make the development of new drug intervention challenging in this population. Translating knowledge about molecular and neurobiological bases into individualized molecular therapies undoubtedly represents the most important long-term challenge for researchers in autism. System Medicine Department, Tor Vergata, University Hospital of Rome, Italy

(e-mail: Curatolo@uniroma2.it).
Copyright $\odot 2017$ by Georg Thieme Verlag KG, Stuttgart · New York
DOI https://doi.org/ 10.1055/s-0037-1603110. ISSN 1304-2580. 